\title{
Unreliability Analysis on the Neutron Detector System Using Monte Carlo Method
}

\author{
Fang Liu ${ }^{1}$ a , Wenjiao Zhu' ${ }^{1}$ and Ping Wang ${ }^{1}$ \\ ${ }^{1}$ Beijing Key Laboratory of Passive Safety Technology for Nuclear Energy, School of Nuclear \\ Science and Engineering, North China Electric Power University, Beijing, 102206, China
}

${ }^{a} E m a i l:$ liuf@ncepu.edu.cn

Keywords: unreliability analysis, neutron detector system, Monte Carlo method Abstract: We use Monte Carlo method to analyze the unreliability performance on the neutron detector system in nuclear reactor. We make the simulation models of three exterior reactor neutron detection channels including source range, middle range and power range measurement channels, and the corresponding three unreliability degrees are calculated. The calculation results show that the unreliability degree of source range and the middle range measurement channel is about in the magnitude of $10^{-6}$ because of their two levels redundancy, and due to four levels redundancy in the power range measurement channels, which unavailability is in magnitude of $10^{-12}$, it has higher safety level than the source and middle range measurement channels.

\section{Introduction}

Unreliability analysis is important to the nuclear reactor safety and especially to the prevention of severe nuclear accidents. The system reliability analysis based on the sense of probability quantification definition can be used to find causes of faults ${ }^{[1]}$. There are two methods to evaluate the reliability of nuclear power plant, one is based on the design criteria of the evaluation method, and the other is the probabilistic safety assessment method (PSA) ${ }^{[2]}$. The determination method has been used in the development of nuclear power plants for a long term. A sensitivity analysis framework based on an incremental Bayesian ensemble updating method is presented to identify the relevant input variables of a severe accident code ${ }^{[3]}$, and a transient identification approach was presented that utilizes clustering for retrieving scenarios information from an Integrated Deterministic and Probabilistic Safety Analysis (IDPSA) ${ }^{[4]}$.The safety margin uncertainties are handled by order statistics to jointly estimate percentiles of the distributions of the safety parameter ${ }^{[5]}$.

The unreliability analysis of the neutron detection system is very important because the neutron detection system provide nuclear reactor power control signal for the safety system. The detector parts distributes in the outer sleeve around the reactor. As the density of neutron flux will change ten orders of magnitude or more from the reactor starting to the normal power working state. Thus, the neutron detection measurement system is divided into three sections, which are the source range channel, the middle range channel and the power range channel to cover the whole measurement range ${ }^{[6]}$. Each measurement range channel uses different detectors to achieve measuring requirement. Two proportional counter tubes are used in source range measurement channel, and two separate ionization chambers are used in middle range measurement channel and four long ionization chambers are used in power range measurement channel ${ }^{[7]}$.

In this paper, we use Monte Carlo method to analyze the unreliability performance of three neutron measurement channels respectively including the source range channel, the middle range channel and the power range channel, and calculated the unreliability degrees which provide reference data for nuclear safety control.

\section{Simulation Models and Methods}

We use Monte Carlo method to simulate a system containing a number of physical units, each unit can be in different states including the work and failure state. In the life of the unit, it can be transferred from one state to another, where the transition time is random. So the stochastic behavior 
of each element can be defined by a matrix that describes the transition probabilities of different states.

In the simulation, we samples at time $t$ based on the conditional probability density of the system $\mathrm{T}\left(\mathrm{t} \mid \mathrm{t}^{\prime}, \mathrm{k}^{\mathrm{s}}\right)$ that is at time $\mathrm{t}^{\text {' }}$ transfering into the state $\mathrm{k}^{\text {' }}$, then sampling at the new state $\mathrm{k}$ based on the the conditional probability density of the system $\mathrm{C}\left(\mathrm{k} \mid \mathrm{k}^{6}, \mathrm{t}\right)$ that is at time $\mathrm{t}$ transfering into the new state $\mathrm{k}^{\text {‘[8] }}$.

In this paper, we analyze the unreliability of the source range, middle range and power range measurement neutron detector system as shown in Fig1. The source range and the middle range neutron measurement systems share two independent measurement channels. So their measrement channel simulation models are same and can be built shown inFig2. We define each detector in the channel as unit $A_{1}$ and $A_{2}$ respectively.

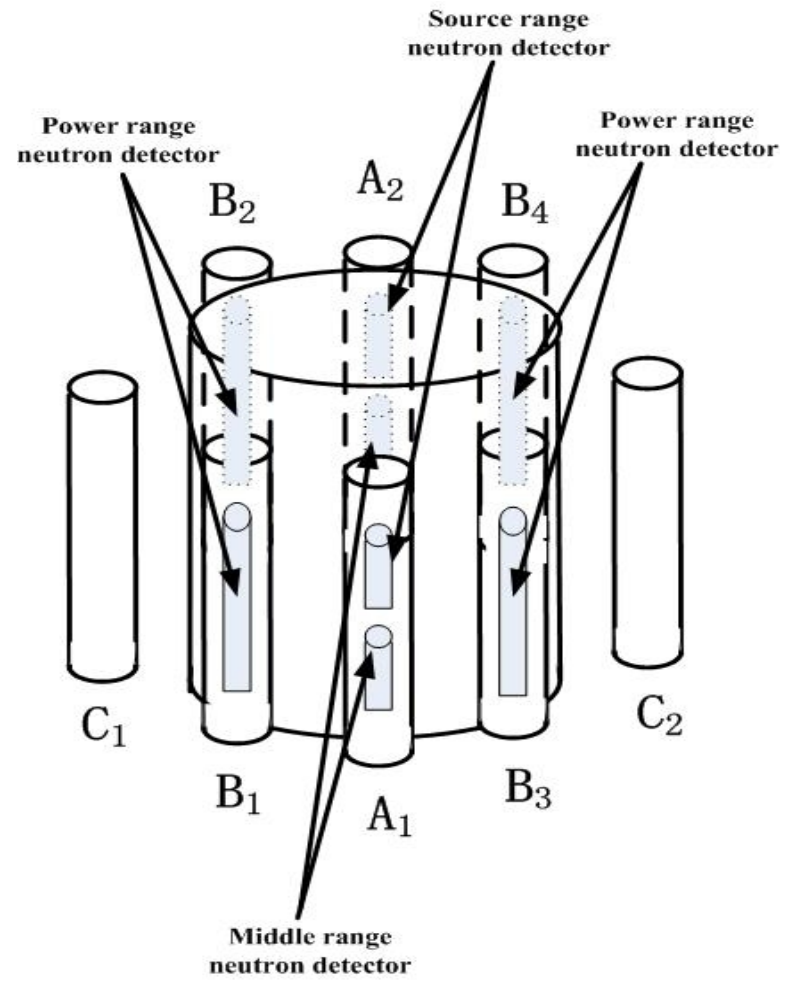

Fig1.The neutron detector channels of pressurized water reactor $\left(\mathrm{A}_{1}, \mathrm{~A}_{2}\right.$ : the source range channels; $\mathrm{B}_{1}, \mathrm{~B}_{2}, \mathrm{~B}_{3}, \mathrm{~B}_{4}$ : the middle range channels; $\mathrm{C}_{1}, \mathrm{C}_{2}$ : the standby measurement channels)

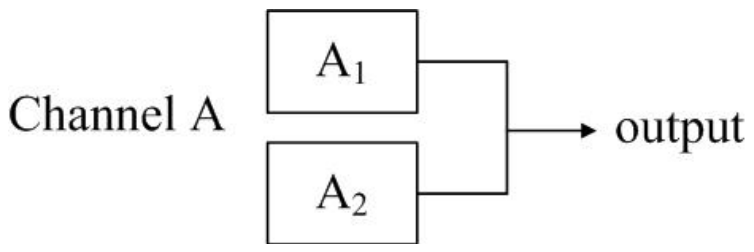

Fig2. The source and middle range channel model

The status of detector includes working ( labeled as 1) and failure (labeled as 2). The initial state is set as 1 . The transition rates are shown in table $1^{[9]}$.

The transfer rate of the system leaving its current configuration state is described as:

$$
\lambda_{1}=1 \times 10^{-4}+1 \times 10^{-4}
$$

We can use the continuous distribution of the inverse transform method to calculated the $t_{1}$ as followed.

$$
\mathrm{t}_{1}=\mathrm{t}_{0}-\frac{1}{\lambda_{1}} \ln \left(1-\mathrm{R}_{\mathrm{t}}\right)
$$

The next transfer rate is: 


$$
\lambda_{2}=1 \times 10^{-1}+1 \times 10^{-1}
$$

So the next time $t_{2}$ is :

$$
\mathrm{t}_{2}=\mathrm{t}_{1}-\frac{1}{\lambda_{2}} \ln \left(1-R_{\mathrm{t}}\right)
$$

Table 1.Transition rate

\begin{tabular}{c|c|c|c}
\hline \multicolumn{2}{c|}{} & \multicolumn{2}{c}{ Arrival Status } \\
\cline { 3 - 4 } \multicolumn{2}{c|}{} & 1 & 2 \\
\hline \multirow{3}{*}{$\begin{array}{l}\text { Initial } \\
\text { Status }\end{array}$} & 1 & 0 & $1 \times 10^{-4}$ \\
\cline { 2 - 4 } & 2 & $1 \times 10^{-1}$ & 0 \\
\hline
\end{tabular}

In the simulation process of random walk process, the system transfers from one state to another one until the time arrivals at Tm, where Tm is set as $1 \times 10^{8}$.

In the source range channel system, when unit $A_{1}$ and $A_{2}$ are simultaneously invalid, the system fails. Through random transfer, we recorded the time of failure and calculated the failure rate by making the failure time divided by the total time. And we got the average unreliability degree by comprehensive multiple random experiments.

The structure of power range measurement channel is given in Fig.1. The model of the power range channels is established with the same method as shown in Fig.3. The power range is composed of four independent measurement channels, and each measurement channel has one long ionization chambers. The measurement channels are labeled as $\mathrm{B}_{1}, \mathrm{~B}_{2}, \mathrm{~B}_{3}$, and $\mathrm{B}_{4}$, and when four channels are simultaneously invalid, the system fails ${ }^{[10]}$.

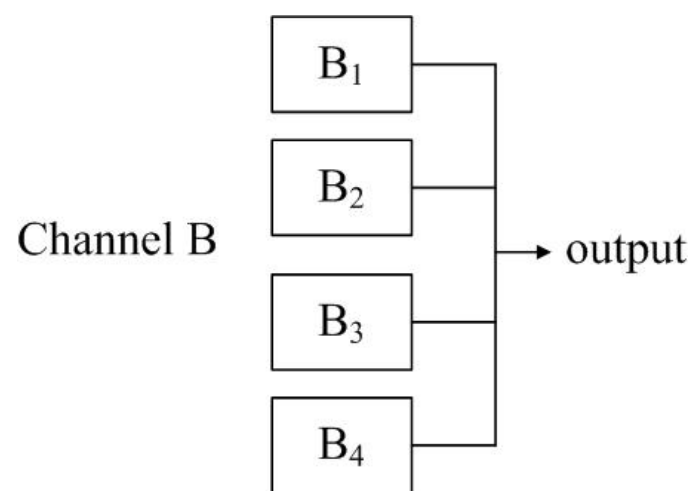

Fig.3. The power range channel model

\section{Simulation Results}

We use the established models (shown in Fig.2 and Fig.3) to simulate the random transfer process and calculate the unreliability of those three neutron detector systems. The simulation results of the source range and middle range channels are shown in Fig.4. It is clear that each experiment is random and the calculated average unreliability degree is $1.0239 \times 10^{-6}$. This result is better than the actual average unreliability of the detector $\left(\sim 1.0 \times 10^{-5[11]}\right)$.

The calculated unreliability result of the power range model is shown in Fig.5. It is clear that the average unavailability degree of the power range channel detector is $3.9498 \times 10^{-12}$. The detector has the magnitude of $10^{-6}$ due to the second level redundancy of the detector due to its secondary level 
redundancy. Because of the fourth level redundancy, the failure probability of the power range measurement channel system has the magnitude of $10^{-12}$ nearly closing to zero.

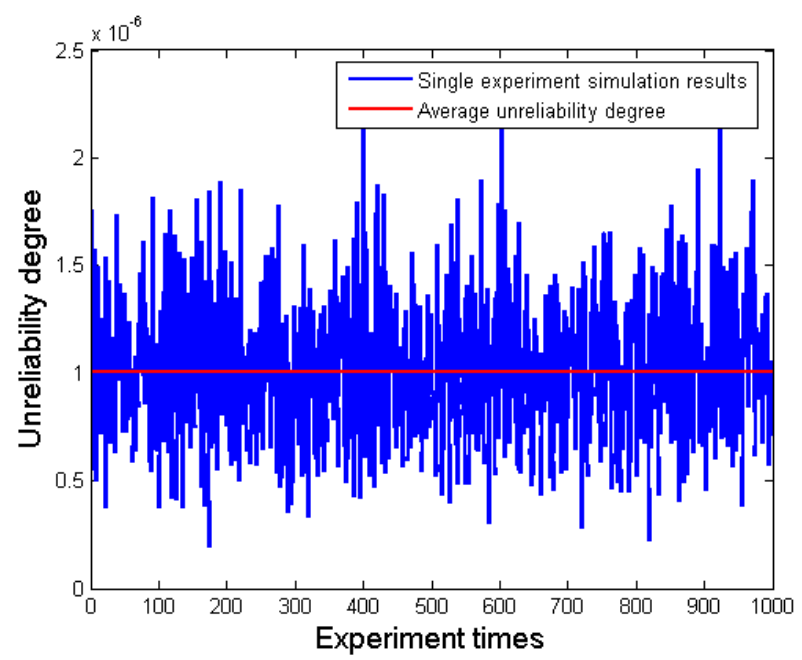

Fig.4. Unreliability simulation results of source range and middle range measurement channels

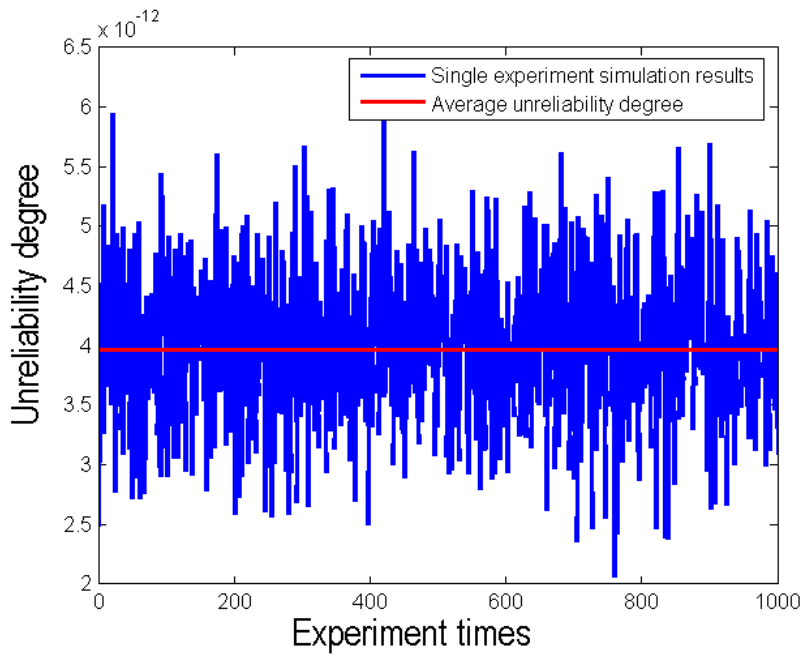

Fig.5 Unreliability simulation result of the power range measurement channel

\section{Conclusions}

In this paper, we analyze the unreliability performance of the neutron detection system in the nuclear power station using Monte Carlo method. In this simulation model, we assume that the neutron detection system should be repaired if one of the detectors is failure. The simulation results show that the calculated unreliability are about in the magnitude of $10^{-6}$ in source and middle range measurement channels and about $10^{-12}$ in power range measurement channel respectively. The calculated unreliability degree is better than the actual statistical data $\left(\sim 1.0 \times 10^{-5}[11]\right)$ because the simulation model is simulated in a ideal model not affected by the actual environment and human factor.

\section{Acknowledgments}

This work was supported by the Project of National Natural Science Foundation of China (Grant No. 11405055) and the Fundamental Research Funds for the Central Universities (Grant No. 2014ZZD09). 


\section{References}

[1] Zhang ZhiGuo, Yang Guang, Gong YingHai, Reliability Analysis of Complex Systems .(Harbin Institute of Technology Press, China 2009)

[2] Zhu JiZhou, Nuclear Reactor Safety Analysis,(Atomic Energy Press, China 2004)

[3] Seyed Mohsen Hoseyni, Francesco Di Maio, Matteo Vagnoli, Enrico Zio, Mohammad Pourgol-Mohammad. A Bayesian ensemble of sensitivity measures for severe accidentmodeling, Nuclear Engineering and Design 295 (2015) 182-191

[4] Francesco Di Maio, Matteo Vagnoli, Enrico Zio, Transient identification by clustering based on Integrated Deterministicand Probabilistic Safety Analysis outcomes. Annals of Nuclear Energy 87 (2016) 217-227

[5] FrancescoDiMaio, AjitRai, EnricoZio, A dynamic probabilistic safety margin characterization approach in support of Integrated Deterministic and Probabilistic Safety Analysis. Reliability Engineering and System Safety. 145(2016)9-18

[6] Zhong Guang He. Introduction to Nuclear Instrumentation System - power system.

[7] Ling Qiu, GuoLanYing Li TongXu Radiation Measurement Technology for Nuclear Power Station. Atomic Energy Press(2001)

[8] Enrico Zio, The Monte Carlo Simulation Method for System Reliability and Risk Analysis. (Springer series in reliability engineering,Springer2013)

[9] China Nuclear Power Plant Equipment Reliability Data Report ,National Nuclear Safety Administration, (2015)

[10]Zhang Hong, Measurement and Treatment of Core Power Distribution in Dayawan Nuclear Power Station. Nuclear Science and Engineering. 1997(1)1-11

[11]Chen JiDong, System and Operation of Dayawan Nuclear Power Station, Atomic Energy Press (1994) 